\section{Cahiers de Narratologie}

Analyse et théorie narratives

Littérature et sciences

\title{
Le Docteur Gregorio Marañón, ou la plume militante de l'endocrinologue
}

\section{Marie-Aline Barrachina}

\section{(2) OpenEdition}

1 Journals

Édition électronique

URL : http://journals.openedition.org/narratologie/5963

DOI : $10.4000 /$ narratologie.5963

ISSN : 1765-307X

Éditeur

LIRCES

Référence électronique

Marie-Aline Barrachina, « Le Docteur Gregorio Marañón, ou la plume militante de l'endocrinologue », Cahiers de Narratologie [En ligne], 18| 2010, mis en ligne le 08 juillet 2010, consulté le 15 novembre 2019. URL : http://journals.openedition.org/narratologie/5963 ; DOI : 10.4000/narratologie.5963

Ce document a été généré automatiquement le 15 novembre 2019.

\section{(c) (i) (9)}

Cahiers de Narratologie - Analyse et théorie narratives est mis à disposition selon les termes de la licence Creative Commons Attribution - Pas d'Utilisation Commerciale - Pas de Modification 4.0 International. 


\title{
Le Docteur Gregorio Marañón, ou la plume militante de l'endocrinologue
}

\author{
Marie-Aline Barrachina
}

Quand on travaille sur l'histoire politique, sociale et culturelle de l'Espagne du premier tiers du XXè siècle, on rencontre nécessairement le Docteur Gregorio Marañón (1887-1960), intellectuel libéral engagé qui joua un rôle de premier plan dans la préparation puis dans la mise en place de la Seconde République (1931-1936). Connu en France pour son essai sur Don Juan et le donjuanisme (1940), le Docteur Gregorio Marañón produisit dans les années vingt du siècle dernier des théories sur la sexualité et sur le genre que les intellectuels organiques du régime franquiste s'empressèrent d'instrumentaliser pour cautionner une politique nataliste drastique et légitimer la relégation des femmes espagnoles dans le domaine privé du foyer. L'opération, il convient de le souligner, fut aisée, car le caractère systématique des analyses du médecin, associé à ses convictions tranchées sur la normalité sexuelle et psychique abondaient dans le sens recherché par le franquisme. Cela n'est pas le moindre paradoxe de ce libéral qui avait réellement pris des risques sous la dictature du Général Miguel Primo de Rivera (1923-1930) et de ce républicain qui a joué un rôle déterminant dans la préparation de l'avènement de la seconde République espagnole (1931-1936), puisqu'il fut de ceux qui préparèrent le pacte de San Sebastián en 1930.

2 Le Docteur Marañón inscrit son œuvre dans le droit fil de la tradition, prégnante dans l'Espagne de la fin du XIXè siècle, des médecins philosophes d'une part, et d'un hygiénisme tardif d'autre part ${ }^{1}$. Ne serait-ce qu'à ce titre, il semble devoir pleinement tenir sa place dans ce volume qui s'interroge sur les liens entre médecine et littérature.

3 Polygraphe compulsif, Marañón a beaucoup publié dans sa spécialité, naturellement, mais il s'est aussi fait un nom en tant qu'intellectuel engagé, et comme historien. Au total, il a à son actif plus de 80 ouvrages, un demi millier d'articles scientifiques, ou de vulgarisation, et des préfaces en grand nombre, puisqu'on en comptabilise environ 220.

Mais par delà cette hyperactivité d'écrivain aussi avide d'histoire et de littérature que de médecine et de politique, ce qui s'avère particulièrement remarquable chez cet auteur, ce qui fait l'objet de cette contribution, c'est que toute son œuvre écrite (œuvre 
d'historien, de publiciste, de vulgarisateur scientifique) est guidée par une conviction qu'il fonde sur ses recherches d'endocrinologue : cette conviction, c'est qu'il existe selon lui une cohérence sans solution de continuité entre la vie physique, la vie psychique et la vie sociale. Guidé par cette conviction, il fait de la médecine, et plus particulièrement de l'endocrinologie, la mesure de bien des choses, et il applique sans trop de scrupules la grille de lecture de l'endocrinologue aux objets d'étude extrêmement variés qui retiennent son attention.

5 Par la médecine, et particulièrement par la spécialité qu'il a faite sienne, Gregorio Marañón cherche en quelque sorte une formule globalisante qui permettrait d'expliquer les comportements humains. Ses premières publications, dès sa thèse de doctorat, sont significatives de cette recherche. Après Investigaciones anatómicas sobre el aparato paratiroideo del hombre (1911), il publie sa conférence prononcée en 1915 à l'Ateneo de Madrid sur La doctrina de las secreciones internas. Su significación biológica y sus aplicaciones a la clínica. Enfin, en 1919, il publie sa première œuvre d'importance sur les effets de la ménopause : La edad critica. Ces travaux et son investissement en tant que praticien à l'Hôpital général de Madrid le conduisent à l'Académie de Médecine où il prononce en mars 1922 un discours de réception intitulé Problemas actuales de la doctrina de las secreciones internas.

Convaincu de «l'intelligence vitale» du système hormonal, Marañón s'engage rapidement dans la défense de ce dernier sur le terrain social, militant à la fois pour une justice sanitaire et pour une reconnaissance - qu'il croit sans tabous, mais nous verrons plus loin qu'il n'en est rien - de l'importance décisive de la libido dans toute activité humaine. C'est dans le droit-fil des courants hygiénistes en plein essor, d'un mouvement international pour la défense du birth control et d'une sexualité assumée, le tout soutenu par les récentes théories sur la concomitance entre les maladies de l'esprit et celles du corps, dont les plus connues sont celles de Freud, que se situe la pensée de Marañón. Il convient en effet de rappeler que Freud a presque tout publié de son oeuvre $^{2}$ quand le jeune docteur Gregorio Marañón, de retour en Espagne après avoir servi comme médecin militaire dans l'Armée française, reprend le service des maladies infectieuses de l'Hôpital général de Madrid pour en faire son Institut de Pathologie médicale, et se lance fébrilement dans sa vie d'homme public et d'intellectuel engagé. Faut-il ne voir qu'un simple hasard dans la similitude entre les titres des trois essais sur la théorie de la sexualité (Freud, 1901) et des tres ensayos sobre la vida sexual (1926) ?

7 Si les coïncidences de la pensée de Marañón avec celles de Freud ne sont plus à démontrer, il n'en faut pas moins souligner la spécificité très "matérialiste » de la pensée de Marañón qui, aussi profondément chrétienne fût-elle, postule la matérialité absolue (chimique) des stimuli. José Luis Abellán résume ainsi la comparaison entre les deux pensées.

Les théories endocrinologiques de Marañón présentent certaines similitudes avec la psychanalyse freudienne, et elles en sont complémentaires: toutes deux s'accordent pour mettre à jour l'idiosyncrasie des individus et pour donner une forme humaniste à la médecine. Comme Freud, Marañón admettait la libido comme impulsion première, mais il considérait que l'énergie libidinale était tout d'abord le résultat d'un phénomène chimique : l'irruption dans le sang des sécrétions internes produites par les gonades; en somme, la contribution endocrinologique du médecin pouvait être considérée comme un support biologique pour les théories freudiennes ${ }^{3}$. 
C'est donc très probablement parce qu'il est convaincu de l'origine chimique (hormonale) de la plupart des comportements humains, que Marañón se lance un jour dans des spéculations sur des types ou des mythes, devenant un militant de sa discipline au service de ses convictions humanistes et sociales.

\section{Le médecin historien}

En France, rappelons-le, on connaît surtout de Gregorio Marañón sa très particulière interprétation de la personnalité donjuanesque. Son ouvrage sur donjuan et le donjuanisme, publié pour la première fois en France en 1958, est le fruit de plusieurs ébauches publiées en Espagne dès 1923, quand il prononce à Santander une conférence sur la «biologie de Don Juan », publiée en 1924 sous le titre Notas para la biología de don Juanet complétée par sa Psicopatología del donjuanismo. Il revint sur don Juan en 1940, au cours de son exil commencé en décembre 1936 et qui s'achève assez rapidement, en 1942.

Dans cet ouvrage, on le sait, Marañón met à mal la virilité de Don Juan dont l'hyperactivité séductrice (plutôt que proprement sexuelle) serait, selon lui, l'indice le plus significatif d'une personnalité à la sexualité inachevée. La thèse est assez connue pour qu'il soit inutile d'y revenir. Elle révèle en tout cas un érudit fin connaisseur des mythes littéraires et des types humains auxquels ils se réfèrent ou qui s'y rapportent (Casanova).

11 Mais pour ce qui nous occupe, cette thèse révèle surtout avec quelle habileté et quelle conviction l'écrivain adopte la grille de lecture de l'endocrinologue comme fil conducteur de son analyse. Croisant les textes et les témoignages, il établit sur Casanova un diagnostic d'immaturité sexuelle - voire d'impuissance - en se fondant sur deux types d'observations: les observations qui touchent aux caractéristiques physiques de son sujet (gigantisme, forme indécise du menton), celles qui touchent à son caractère, révélé dans le cas précis par les mémoires, que Marañón juge tout à fait symptomatiques d'un exhibitionnisme pathologique.

12 Il importe de souligner que cette tentative d'interprétation médicale d'un type humain (ici emprunté à la littérature nationale) n'est que la première d'une série dans la bibliographie de Marañón, qui abandonne bientôt le mythe littéraire auquel il préfère, on le voit déjà avec le cas de Casanova, le personnage historique qui lui permet de se livrer à ce qu'il appelle de "l'archéologie médicale ${ }^{4}$ ", autrement dit du diagnostic médical historique.

13 Membre de l'Académie royale de Médecine depuis 1922, on l'a vu, le docteur Marañón s'adonne avec passion à la recherche historique et plus particulièrement dans le domaine de la biographie de certaines personnalités de l'histoire nationale chez lesquelles il décelait des traits de caractère ou de comportement susceptibles d'être éclairés par le diagnostic médical ou de fournir du matériel pour une étude de cas.

Il l'affirma sans ambages dans son prologue à la première édition de son Ensayo biológico sobre Enrique IV de Castilla y su tiempo ${ }^{5}$. Il y écrivait que si l'on peut être à la fois médecin et historien, ce n'est pas la compétence de l'historien mais celle du médecin qui pouvait, dans le cas d'Henri IV, apporter un éclairage nouveau et résoudre le mystère de la naissance de la « Beltraneja $^{6} »$. 

historiques $^{7}$, diagnostique chez Enrique IV de Castilla (1425-1454-1474) une impuissance partielle liée à une "psychologie schizoïde » dérivée des caractéristiques morphologiques du monarque. Le diagnostic se fonde sur l'analyse des chroniques de l'époque, en particulier celles d'Alonso de Palencia et de Hernando del Pulgar. Il s'appuie en outre sur une demi-douzaine de portraits du monarque déchu. L'observation des portraits et l'étude des témoignages conduisent Marañón à déceler sur la personne d'Henri les symptômes de l'acromégalie décrite en 1885 par un certain Pierre Marie (1853-1940), maladie invalidante qui aboutirait, à terme, à une impuissance secondaire.

Plus tard, en 1932, l'étude de la biographie d'Amiel (Henri Fréderic, 1821-1881) permet de nouveau à Marañón d'établir une relation entre caractéristiques physiologiques et traits de caractère. C'est la publication de Amiel, un estudio sobre la timidez, qui sera suivi de plusieurs autres ouvrages biographiques orientés de cette façon, et dont les plus importants sont $\mathrm{El}$ Conde Duque de Olivares, la pasión de mandar (1936) une étude de cas sur l'ambition, Tiberio, historia de un resentimiento (1939) etc. Les premiers de ces travaux valurent à notre auteur d'être reçu, en pleine seconde République, à l'Académie de la Langue espagnole (1934) puis à l'Académie d'Histoire (1936).

\section{De l'université à l'engagement : toujours le médecin}

17 Né en 1887, Gregorio Marañón avait soutenu sa thèse de doctorat en médecine en 1910 à l'âge de 23 ans. Après un bref séjour à Vienne où il avait rencontré Freud, il s'était rendu en Allemagne où il étudia auprès de l'endocrinologue Ehrlich (post doctorat). Il obtint à son retour en 1911 un poste à l'hôpital Général de Madrid (service des maladies infectieuses) et épousa dans la foulée la fille du directeur du journal El Liberal. Il confirmait de la sorte son appartenance au milieu intellectuel et libéral dans lequel il évoluait depuis l'enfance: son père, un éminent juriste, avait pour amis l'historien Marcelino Menéndez Pelayo et les écrivains Benito Pérez Galdós et José María Pereda.

S'il ne tarda guère à publier les résultats de ses recherches de spécialiste, c'est seulement après son séjour sur le Front en France où il étudia en mission officielle la grippe espagnole qu'il entama une véritable carrière de publiciste et de vulgarisateur de ses propres recherches en endocrinologie.

19 En 1919, il commence à publier dans la presse généraliste (El Debate, El Liberal) et donne au public, sous le titre La edad crítica, la première version d'un ouvrage qui lui tient à cœur et qu'il remanie plusieurs fois avant de lui donner sa version définitive sous le titre El climaterio de la mujer y del hombre (1937).

Dans le contexte difficile du début des années vingt, où l'Espagne peine à accéder à la modernité, le docteur Gregorio Marañôn s'insurge contre les conséquences sanitaires de la pauvreté. En juin 1922, peu après son entrée à l'Académie royale de Médecine (mars 1922) il entraîne le monarque Alphonse XIII dans un voyage dans la région la plus déshéritée d'Espagne, «Las Hurdes»-dont la misère a été immortalisée par le documentaire de Buñuel Terre sans pain (1932).

21 En 1926, dans le deuxième de ses " trois essais sur la vie sexuelle » intitulé « maternidad y feminismo ", il se prononce clairement, en tant que médecin et endocrinologue, en faveur du contrôle des naissances et de l'éducation des femmes en ce sens ; il dénonce 
violemment la surmortalité infantile qui découle de la surnatalité, un gaspillage humain insupportable; il stigmatise l'hypocrisie des politiques natalistes et belliqueuses...

Intellectuel libéral engagé sur le terrain social, le Docteur Marañón l'est bel et bien, au point d'être poursuivi sous la dictature de Primo de Rivera (on le soupçonne d'avoir participé à la tentative de coup d'état de juin 1927), et il l'est parce que ses convictions scientifiques l'y engagent : il multiplie les conférences sur la sexualité et soutient de sa notoriété tout ce courant de juristes, de médecins, d'écrivains libéraux qui militent en faveur du birth control et d'une sexualité assumée. On retiendra qu'en 1928 les "premières journées sur l'eugénisme " essentiellement consacrées à la question du contrôle de la natalité et organisées dans les locaux de l'université San Carlos de Madrid par la Gaceta Médica Española, furent suspendues quelques jours avant la conférence très attendue de Marañón, au prétexte très officiel que ces journées n'étaient qu'un "divertissement pornographique » (note circulaire du ministre de l'Instruction publique, 20 mars $\left.1928^{8}\right)$.

\section{La normalité selon le docteur Marañón}

On l'a bien vu, le Docteur Gregorio Marañón met sa plume d'endocrinologue au service de la recherche historique, il met sa plume d'endocrinologue au service du droit des femmes au contrôle de leur natalité, au service de la lutte contre la misère sociale... Mais ce faisant, il met en définitive sa plume au service d'une conception extrêmement rigide de ce qu'il juge être la normalité.

Marañón croyait fermement que le système hormonal régissait l'ensemble des comportements humains et que la normalité sociale dépendait directement d'une normalité que l'endocrinologue était habilité à définir.

\section{Le climat d'inquiétude des années 20}

C'est dans cette perspective qu'il s'autorise, en sa qualité de médecin, à tracer les contours de la normalité sexuelle et de ses "caractères secondaires ", répondant ainsi de façon rassurante à l'inquiétude née d'une sorte de brouillage des normes et des codes sociaux : le féminisme devient depuis la fin de la première guerre mondiale un phénomène social avec lequel il faut compter en Espagne : la mode des cheveux courts, des vêtements plus confortables, la mixité qui se banalise dans les lieux publics, le tout associé à la vulgarisation des thèses sur la bisexualité (auxquelles adhère Marañón), ont contribué grandement au développement de la crainte d'un effondrement social et culturel lié à l'indifférenciation sexuelle ou à la «virilisation» des femmes ${ }^{9}$. On peut lire couramment dans les années 20 et 30 des propos comme ceux que tient le Docteur Roberto Novoa Santos :

Jusqu'au moment présent, tout ce que nos pouvons voir de notre passé nous démontre que l'évolution sexuelle de l'Humanité s'est faite dans le sens d'une plus grande différenciation sexuelle. La femme est devenue de plus en plus femme, et l'homme de plus en plus homme ... mais aujourd'hui, messieurs, ou dans un futur proche ou lointain, que va-t-il se passer, que va-t-il nous arriver?

[...] La femme est de plus en plus forte, de plus talentueuse ; cette femme géniale, encyclopédique, l'est précisément pour ce qu'elle a un peu d'homme en elle [...] de 
sorte que la femme se surpasse parce qu'en elle c'est le mâle agressif et combattif qui l'emporte.

26 Face à cette inquiétude, les théories se multiplient, et le corps médical - qui depuis un siècle déjà avait pris l'habitude d'intervenir dans les débats de société - se mobilise pour endiguer le flux et fixer de nouvelles normes susceptibles de rétablir les fondements d'une différenciation sexuelle des rôles sociaux tout en intégrant les nouveaux codes imposés par la modernité. Certainement pas seul dans cette bataille, le Docteur Gregorio Marañón y tient néanmoins un rôle de premier plan en raison de son prestige.

\section{Les normes morphologiques de la virilité selon Marañón}

27 On a bien compris, à travers les exemples de Don Juan et d'Henri IV de Castille, que le Docteur Gregorio Marañón a une conception très précise de la normalité en ce qui concerne la virilité. Dans son don Juan, il affirme que le «type hyper génital » est "caractérisé précisément par la taille exiguë et le prognathisme», concluant sans transition par un argument parfaitement antiscientifique et hautement littéraire: «C'est ainsi que le satyre est toujours représenté, même dans les caricatures populaires ${ }^{10} »$.

On voit ici le grossier mécanisme d'une argumentation qui croise une observation statistique qui vaut ce qu'elle vaut et une croyance populaire qui vaut aussi ce qu'elle vaut, pour en faire une vérité scientifique. On observe dans le Enrique IV... des raccourcis du même type, très révélateurs des critères idéologiques qui président aux conclusions du Docteur Gregorio Marañón : dans son souci de légitimer l'intromission médicale dans le débat sur la personnalité de Henri IV de Castille, Marañón n’hésite pas à établir sans autre forme de procès des équations peut-être hasardeuses. Non content d'associer à une pathologie physiologique invalidante (l'acromégalie), et à une pathologie psychique lourde (la schizophrénie) l'impuissance et l'homosexualité, Marañón complète le tableau clinique du monarque par un certain nombre de remarques desquelles il ressort une volonté délibérée de classer comme pathologiques des caractères morphologiques plus ou moins accentués liés au rythme de la croissance et au déclenchement plus ou moins précoce de la puberté.

Selon Marañon, point de sexualité épanouie hors de la norme méditerranéenne des pubertés précoces. Suivant sans états d'âme les chroniqueurs contemporains de Henri IV, Marañón admet en effet l'âge de douze ans comme âge de la plénitude sexuelle, et ne s'interroge pas sur le fait que le médecin du jeune prince ait fait état de manifestations d'impuissance dès l'âge de 12 ans. Plus loin, Marañón signale le caractère inhibiteur de la cérémonie nuptiale, mais loin d'en tirer un argument en faveur du jeune roi, il en tire au contraire confirmation de cette "timidité sexuelle » liée selon lui à l'obsession de son "infériorité sexuelle ». À aucun moment, en fait, Marañón n'envisage l'impuissance sexuelle du roi comme une impuissance évolutive liée à une croissance difficile et à une pression sociale forte. S'il reconnaît l'existence de l'une et de l'autre, c'est à une pathologie spécifique du roi qu'il attribue l'incapacité de ce dernier à les surmonter victorieusement. Autrement dit, derrière le discours médical se cache un discours idéologique résolument "machiste ", qui mesure la norme virile à l'aune de la norme sociale établie. Aussi ne s'étonnera-t-on pas de trouver dans les 
différentes approches diagnostiques de Henri IV un chapelet d'étiquettes fondées sur une perception rigide de la virilité.

30 En bref, la norme de la sexualité masculine selon le Docteur Gregorio Marañón se confond avec la norme statistiquement contestable de la physiognomonie méditerranéenne.

\section{Caractères sexuels secondaires et comportements sociaux}

31 On a indiqué précédemment que, préoccupé de différenciation sexuelle comme le plupart de ses contemporains, le docteur Marañón prétendait dessiner les contours de la normalité et de ses "caractères secondaires ». Dans l'un de ses ouvrages les plus connus et les plus lus, intitulé Essais sur la vie sexuelle, Marañon s'essaie à la définition de ces «caractères secondaires ». Dès les premières pages du premier de ces essais, on comprend le sens que donne Marañón à la notion de "caractères secondaires ", et on comprend surtout à quel point est déterminant chez lui son aspiration à éclairer les relations sociales à la lumière de ses observations de médecin. On regrettera néanmoins que l'essayiste l'emporte ici sur le scientifique, et que le médecin ne parvienne pas à se soustraire aux préjugés de l'homme soumis aux valeurs libérales de son temps.

Qu'on en juge : dans cet essai qui s'intitule Sexo, trabajo y deporte, Marañón s'interroge tout d'abord sur les raisons pour lesquelles les hommes travaillent. Après avoir écarté l'interprétation biblique, il s'empresse d'affirmer que le travail répond à une nécessité physiologique et qu'il est, en quelque sorte, une fonction d'ordre sexuel ${ }^{11}$. A l'aide d'un tableau qui tente de conférer un caractère systématique et rationnel à la démonstration, Marañón rappelle que femmes et hommes se différencient par des caractéristiques anatomiques et fonctionnelles, primaires et secondaires. Il ajoute que la fonction primaire génitale féminine a pour corollaire des fonctions secondaires inéluctables telles que l'instinct maternel, une plus grande sensibilité affective et une faible disposition pour les activités créatrices, une démarche et des attitudes caractéristiques et une voix aiguë. Parallèlement, la fonction génitale masculine aurait pour corollaire un instinct poussant vers l'action, une plus faible sensibilité affective et une plus grande aptitude pour l'abstraction et la création, ainsi qu'une démarche et des attitudes caractéristiques et une voix grave. Tout un détour par l'observation clinique pour aboutir à la consternante confirmation du bien-fondé d'un ordre patriarcal pluriséculaire! Et pour conclure

On voit donc bien que le travail est directement lié au sexe, comme activité masculine complémentaire et parallèle de l'activité féminine de la maternité. L'analyse biologique confirme ainsi le sens profond de la première page de la genèse : Adam naît pour le travail au moment même où Eve, notre mère à tous, naît pour la vie entièrement liée au sexe. Et Dieu leur indique à chacun très clairement leurs deux chemins parallèles: «Toi, l'homme, tu travailleras; toi, femme, tu enfanteras ${ }^{12}$ ».

Sur ces bases, Marañón s'emploie tant dans ses travaux d'endocrinologie que dans ses ouvrages de vulgarisation à dessiner au plus près les types biologiques parfaits de la virilité et de la féminité, dont le développement garantirait l'harmonie sociale.

Certes, ce faisant, il va à l'encontre du tabou de la sexualité, puisqu'il revendique la sexualité féminine comme une nécessité vitale. Mais il construit - ou reconstruit plutôt, revêtu d'une légitimité nouvelle garantie par la science - un modèle comportemental d'une rare inflexibilité. 
nd il évoque - toujours avec bienveillance - tel patient victime d'une attirance homosexuelle plus ou moins avérée, ou telle patiente chez laquelle il a décelé certaine forme de frigidité, c'est toujours pour mettre en cause une " anomalie » morphologique ou hormonale. La "déviance » qu'il faut soigner coûte que coûte vient remplacer le " péché » qu'il fallait combattre.

\section{Adaptation et récupération}

Dès lors on ne s'étonnera pas d'un retour en grâce relativement précoce pour un libéral qui fut un ouvrier de la Seconde République: ses thèses sur la sexualité féminine étaient en effet promises à un brillant avenir dans un régime nataliste et soucieux de renvoyer les femmes dans leurs foyers. Gregorio Marañón n'avait pas eu à choisir au moment où éclata la guerre civile. Contrairement à Miguel de Unamuno, présent dans son université de Salamanque dont il était le Recteur, et qui se fourvoya dans le soutien au soulèvement militaire au nom de la préservation de l'ordre républicain, contrairement à Antonio Machado, résolument républicain quoi qu'il en coûtât, Gregorio Marañón, comme le philosophe Ortega y Gasset, était absent en juillet 1936. L'un et l'autre optèrent pour un exil mutique. Marañón put regagner l'Espagne dès 1942. Il se consacra dès lors à ses études historiques, mais ses travaux en matière de sexualité féminine et en matière d'endocrinologie furent largement utilisés par les médecins du franquisme. Son élève, le docteur José Botella Llusiá, ainsi que le docteur Francisco Luque Beltrán, qui écrivaient régulièrement dans la presse féminine dans les années 40 , se réclament systématiquement des travaux de Marañón pour rappeler la norme et inciter les lectrices à travailler leur corps et leurs esprits à la recherche d'une féminité patriotique sans faille. L'image de Marañón reste associé à un libéralisme de bon aloi, à un républicanisme modéré et à un humanisme social incontestables qui n'occultent pas tout à fait, néanmoins, le caractère ambigu d'une pensée fortement volontariste.

\section{NOTES}

1. José Luis Abellán, Historia crítica del pensamiento español, vol. 5, La crisis contemporánea, (1875-1936), Espasa Calpe, Madrid, 1989.

2. Il manque Essai de psychanalyse (1927), Malaise dans la civilisation (1930) et Moïse et le monothéisme (1939).

3. In Abellán, Historia crítica ... p. 317.

4. Ensayo biológico sobre Enrique IV de Castilla y su tiempo, por G. Marañón, de las Academias Españolas de Medicina y de Historia, Segunda edición aumentada, Espasa Calpe S. A., Madrid, 1934, chapitre 1, p. 21.

5. Première publication en 1930 ; deuxième publication augmentée et remaniée, en 1934. La publication de 1946, survenue après l'exhumation et l'examen médical de la dépouille d'Henri IV de Castille ne subira pas de modification par rapport à l'édition de 1934, cet examen ayant confirmé les observations du Médecin biographe.

Cahiers de Narratologie, 18 | 2010 
6. Le doute sur la légitimité de Jeanne, fille unique d'Henri de Castille, suspecté d'impuissance, a été un élément déterminant dans la prise du pouvoir par Isabelle de Castille dite « la catholique ». La jeune princesse fut très rapidement affublée du sobriquet de Beltraneja, allusion plus que marqué au nom de l'amant supposé de la reine, Beltrán de la Cueva

7. - 1930 : Enrique IV ; - 1932 : Amiel, un estudio sobre la timidez; - 1934 : Las ideas biológicas del padre Feijoo ; - 1936 : El Conde-duque de Olivares, la pasión de mandar ; - 1939 : Tiberio, historia de un resentimiento ; - 1940 : Don Juan ; - 1942 : Luis Vives ; - 1947 : Españoles fuera de España ; - 1947 : Antonio Pérez ; - 1950 : Ramón y Cajal y su tiempo ; - 1957 : El Greco y Toledo ; - 1960 : Los tres Vélez.

8. Sur ces journées de 1928 et celles de 1933, voir, Marie-Aline Barrachina, Las primeras jornadas eugénicas españolas (Madrid 1928-Madrid 1933), Hommage à Nelly Clemessy, Publications de la Faculté des lettres, arts et sciences humaines de Nice, Université de Nice-Sophia Antipolis, $\mathrm{n}^{\circ} 14$, nouvelle série, Nice, 1993, p. 43-55. Et Maternidad, feminidad, sexualidad. Algunos aspectos de las «Primeras jornadas eugénicas españolas, Madrid 1928-Madrid 1933, Hispania, Revista española de Historia, Sección monográfica. La sexualidad en la España contemporánea, coord. J.-L. Guereña, septiembre-Diciembre 2004, vol LXIV/3, 218, CSIC, Madrid, p. 1003-1026.

9. Pour une analyse précise et détaillée de ce phénomène en Espagne, voir Nerea Aresti, Médicos, Donjuanes y Mujeres modernas, Los ideales de feminidad y masculinidad en el primer tercio del siglo XX, Universidad del País Vasco, Bilbao, 2001.

10. Gregorio Marañôn, Don juan et le Donjuanisme, version française, Gallimard, idées, 1958, p. 181.

11. Marañón, Gregorio, œuvres complètes, Espasa Calpe, Madrid, vol VIII, 1966, p. 268.

12. Ibid. p. 274.

\section{RÉSUMÉS}

Jouissant dès le début des années vingt d'un grand prestige dû à ses travaux sur les glandes endocrines, le Docteur Gregorio Marañón (1887-1960) s'engagea de tout son poids dans le débat social, mettant son autorité de médecin reconnu au service de la lutte contre la misère. Dans cette même perspective, c'est en endocrinologue convaincu de « l'intelligence vitale » du système hormonal, qu'il prit position en faveur de la limitation des naissances et de la reconnaissance de lasexualité féminine, dans un contexte où cette question était au cœur des conflits idéologiques qui traversaient la société espagnole entre 1920 et 1936. Porté par un important courant de juristes, de médecins, d'écrivains libéraux qui militaient en faveur du birth control et d'une sexualité assumée, le prestigieux docteur Marañón apportait sa légitimité scientifique à la démarche. Or, les thèses de Marañón, promises à une longue et faussement paradoxale postérité sous le franquisme, reposent sur un double postulat qui affirme une certaine forme de déterminisme biologique des comportements humains, et qui affirme aussi, à l'instar des théories freudiennes, l'importance décisive de la sexualité dans ces comportements. On tentera de montrer comment les théories de l'endocrinologue libéral ont pu, dans les années 40, être instrumentalisées pour apporter leur caution scientifique à la morale sexuelle imposée par le franquisme. Les théories de Marañón ont en effet fourni aux intellectuels organiques du régime franquiste tant d'arguments pour sa politique nataliste et pour la relégation des femmes dans le domaine privé du foyer que ses écrits sont incontournables pour quiconque se penche sur cet aspect de l'histoire de la dictature. 
El Doctor Gregorio Marañón (1887-1960), que gozaba en los años veinte de un gran prestigio debido a sus trabajos sobre endocrinología, se comprometió sin reparos en el debate social, y puso su autoridad de médico al servicio de la lucha contra la miseria. Como endocrinólogo convencido de la « inteligencia vital » del sistema hormonal, se declaró claramente favorable al birth control y al reconocimiento de las exigencias de la sexualidad femenina, en un contexto que hacía de estas cuestiones unas cuestiones claves de los conflictos ideológicos del momento en la sociedad española (años 1920-1936). El prestigioso Doctor Marañón aportaba su aval científico a un amplio movimiento de juristas, médicos, escritores libérales favorables al birth control y a una sexualidad femenina asumida. Ahora bien, las tesis de Marañón, prometidas a un largo porvenir bajo el régimen de Franco, tienen su fundamento en un doble postulado que afirma al mismo tiempo cierto determinismo biológico de las pautas humanas de comportamiento, y el peso decisivo de la sexualidad para la definición de dichas pautas. Se intentará mostrar cómo las teorías del endocrinólogo han podido ser instrumentalizadas por los intelectuales orgánicos del régimen de Franco para aportar un aval científico a la moral sexual del franquismo. Las teorías de Marañón proporcionaron tantos argumentos para dicha moral y para la subsiguiente relegación al hogar de las mujeres españolas que esos escritos son de inexcusable estudio a la hora de analizar la cuestión del género en la dictadura de Franco.

\section{INDEX}

Index chronologique : XXe siècle

Index géographique : Espagne

Mots-clés : autorité, endocrinologie, idéologie, limitation des naissances, norme sexuelle, sexualité

\section{AUTEUR}

\section{MARIE-ALINE BARRACHINA}

Université de Nice Sophia Antipolis, Centre de la Méditerranée Moderne et Contemporaine, (EA 1193) 